\title{
ASSESSMENT OF PATHOGENICITY OF SOIL FUNGI TRICHODERMA VIRIDE 505
}

\author{
N. O. Kravchenko, Ye. P. Kopylov, O. V. Holovach, O. M. Dmytruk
}

World practice proves the existence of the trend of increasing crop losses resulting from the activity of harmful organisms. This cannot be prevented by the use of chemical plant-protection agents and disease-resistant crop varieties. Therefore, many developed countries have urgent need for decreasing of pesticide use because of negative phenomena arising from their widespread use.

The above leads to the search for new methods of protecting plants against diseases that are more advanced than traditional. An integrated plant protection was and remains an alternative to a chemical method, an important component of which is a biological method, in particular, the use of microbial preparations. Microbial preparations do not pollute the environment, show high selective effect, and are suitable for production. When they are used, crop products do not contain pesticide residues, chemical load on the environment decreases.

The representatives of widespread soil micromycetes of Trichoderma Pers. genus cause the greatest interest among bio-agents of microbial preparations for protection of plants from root disease pathogens. Trichoderma genus fungi are characterized by antibiotic and antagonistic properties.

An antagonistic effect of Trichoderma genus fungi is subject to its ability to form antibiotics (gliotoxin, viridin, alamecin etc.), hydrolytic enzymes, and actively compete in the development of nutrient substrate and show biparasitic activity [1-3].

Works, devoted to the study of antagonistic properties of this fungi genus and the efficiency of their use as plant protection remedies from pathogens, have a long history. It was established that Trichoderma lignorum and $T$. harzianum inhibit the development of Fusarium oxysporum, $F$. moniliforme, $F$. roseum, $F$. solani and Bipolaris sorokiniana both in the laboratory and in field experiments. Adding of these antagonists into the soil contributes to a significant restriction of root rot of spring wheat, spring barley, cotton, melons $[4 ; 5]$.
The data obtained by British researchers [6] prove the antagonistic activity of Trichoderma species as for phytopathogenic fungus Pseudocercosporella herpotrichoides. The authors note that among the studied micromycetes-antagonists belonging to different genera, the representatives of Trichoderma genus most actively inhibited the growth of the pathogen. The conducted studies are of interest because pathogen

Pseudocercosporella herpotrichoides (Fron.) Deighton (known as Cercosporella herpotrichoides Fron. until 1973) is a causative agent of Cercosporella rot of barley and other grains [7], and causes blackening and dying of roots, and coleoptile.

Antagonistic relationships occur between Trichoderma harzianum and phytopathogenic fungi of Pythium genus. Thus, experiments conducted in Israel, convincingly proved that $T$. harzianum bioagent sharply limited the development of root rot of peas, cucumbers, tomatoes and peppers. Biological efficacy of this antagonist against the said disease was 76-88\% [8].

Antagonistic activity of Trichoderma is detected during the interaction with Sclerotium rolfsii - a causative agent of charcoal rot of sugar beet, Gaemannomyces graminis - a causative agent of root rot of barley and other spring crops. An antagonism in this case is subject to biparasitic activity and formation of toxins, antibiotics [9].

Noteworthy are the works, which reveal the mechanism of action of Trichoderma species. Thus, the antagonistic effect of Trichoderma spp. as for phytopathogenic fungi Pythium sp., which together were grown in a Petri dish, is explained by the factor that caused cells plasmolysis of hyphae tips of Pythium sp. before osculation with an antagonist [10]. In this case, the authors dealt with volatile antibiotic substances, many studies with which were conducted by V. Y. Bilai [2]. It was also established that Trichoderma spp. is characterized by chemo-tropical reaction and the ability to recognize phytopathogenic fungi at a distance. Apparently, lectins play an 
important role in the interaction of host and parasite cells. An antagonist begins to produce lytic enzymes only after the recognition and attachment to the cell wall of the fungus host. Antagonist enzymes are able to decompose the polymers contained in the cell walls of pathogenic fungi. The existence of active chitinase and $\beta$-glucanase in the soil inhabited by fungi Trichoderma genus may be some evidence to this [11].

Search of active strains of fungi of Trichoderma genus - potential agents of biological preparations, is carried out in many academic institutions. One of the requirements for future strains is that along with high antagonistic activity against plant pathogens, they should be safe for humans and warm-blooded animals.

The goal of our work was to study the pathogenic properties of soil fungus Trichoderma viride Pers 505, which is characterized by high antagonistic and antibiotic activity and can be the basis for a biological preparation for protection of plants from pathogens.

Materials and methods. Fungus $T$. viride Pers 505 was grown for 7 days on a wort agar with mass fraction of solids of $3.5 \%, \mathrm{pH} 7.0$ at the temperature of $27^{\circ} \mathrm{C}$. Spore-mycelial fungus mixture was used for the study. The homogenized suspension of spore-mycelial fungus mixture for administration in animals was prepared on a sterile isotonic sodium chloride mixture followed the preliminary two-time washing off metabolites and sedimentation of cells by centrifugation for $20 \mathrm{~min}$. at $2000 \mathrm{rpm}$. To prepare the use rates of fungus cells in suspension Goriaiev chamber was used.

Testing of pathogenic properties of soil fungus strain was performed in acute experiments on white mice models. Outbred mature mice weighing 18-20 g orally received spore-mycelial fungus mixture through a gastric tube and intraperitoneally through injection. For oral administration doses of $1.0 \times 10^{6} ; 4.0 \times 10^{7} ; 1.0 \times 10^{8}$ cells per mouse were used, and for intraperitoneal injection doses of $1.0 \times 10^{7}$ and $1.0 \times 10^{8}$ cells per mouse [12]. Mice were pre-conditioned to husbandry for 14 days. Care and follow-up over the animals were carried out daily within 20 days after the administration of the study material $[12 ; 13]$.
At the end of follow-up over the behavioural reactions and physiological condition of mice they were sacrificed, with subsequent autopsy, microscopic studies of impression smears of internal organs and inoculation of tissue samples (liver, kidney, spleen) onto elective digest medium.

Keeping, feeding, grooming and all manipulations with laboratory animals were conducted under the European Convention for the Protection of Vertebrate Animals Used for Experimental and Other Scientific Purposes (Strasbourg, 1986) [14] and General ethic principles of animal experimentation adopted by the First National Congress of Bioethics (Kyiv, 2001) [15]. Experiments were conducted in compliance with the principles of humanity given in the European Union Directive [16].

The virulence, which is generally accepted conditional measure of pathogenicity, of fungus studied strains was established by the level of the dose of viable microbial cells that causes death in $50 \%$ of infected animals $\left(\mathrm{LD}_{50}\right)$. The criterion of avirulence of a microorganism was absence of death in mice within 20 days and specific for infectious diseases changes in the internal organs of animals at autopsy.

The infectivity (invasiveness) of strain was estimated by determining the capability of the microorganism cells for the dissemination into the tissues of internal organs of animals after a single oral administration (per os) of suspensions of an investigated fungus into the macroorganism. Absence of growth of the fungus on wort agar after inoculation of the samples of internal organs pointed to non-infectivity (noninvasiveness) of an investigated microorganism.

Therefore, both oral and intraperitoneal administration of the suspension of alive cells of Trichoderma viride Pers 505 strain do not lead to bacteria invasion into the internal organs of animals. Pathological and bacteriological study of internal organs of experimental animals have shown that Trichoderma viride Pers 505 is not infective, does not either disseminate nor reproduce in warm-blooded animals. The avirulence of fungus strain for warm-blooded animals is confirmed by average lethal doses 
for both oral administration and intraperitoneal injection.

Thus, according to the obtained results, Trichoderma viride Pers 505 belongs to the group of avirulent microorganisms, not capable to invade internal organs of studied warm-blooded animals and can be considered as non-pathogenic. 